\title{
High pseudocapacitive cobalt carbonate hydroxide films derived from CoAl layered double hydroxides $\uparrow$
}

\author{
Zhiyi Lu, ${ }^{a}$ Wei Zhu, ${ }^{a}$ Xiaodong Lei, ${ }^{a}$ Gareth R. Williams, ${ }^{b c}$ Dermot O'Hare, ${ }^{b}$ Zheng Chang, ${ }^{a}$ Xiaoming Sun $* a$ \\ and Xue Duan ${ }^{a}$
}

Received 14th March 2012, Accepted 18th April 2012

DOI: 10.1039/c2nr30617d

\begin{abstract}
A thin nanosheet of mesoporous cobalt carbonate hydroxide (MPCCH) has been fabricated from a CoAl-LDH nanosheet following removal of the Al cations by alkali etching. The basic etched electrode exhibits enhanced specific capacitance $\left(1075 \mathrm{~F} \mathrm{~g}^{-1}\right.$ at $\left.5 \mathrm{~mA} \mathrm{~cm}^{-2}\right)$ and higher rate capability and cycling stability $(92 \%$ maintained after 2000 cycles).
\end{abstract}

Supercapacitors (SCs), also called electrochemical capacitors, are currently receiving intense attention as potential candidates for use in portable electronics, in hybrid electric vehicles (EV) and for industrial scale power production because they possess a number of attractive properties (e.g. high power density and long cycle life). ${ }^{1,2}$ Generally, SCs store energy either using ion adsorption (electrochemical doublelayer capacitors, EDLC) or by fast surface redox reactions (pseudocapacitors). In order to improve the specific capacitance of SCs, which is essential for practical usage, substantial effort has been focused on investigating the pseudocapacitive performance of transition metal oxides and hydroxides (e.g. $\mathrm{Ni}(\mathrm{OH})_{2}, \mathrm{Co}(\mathrm{OH})_{2}$, $\left.\mathrm{MnO}_{2}\right) \cdot^{3-5}$ These materials produce capacitances exceeding those of double-layer carbonaceous materials such as carbon nanotubes ${ }^{6}$ and graphene. $^{7}$

Layered double hydroxides (LDHs) are layered anionic clays that are generally expressed by the formula $\left[\mathrm{M}^{2+}{ }_{1-x} \mathrm{M}^{3+}{ }_{x}(\mathrm{OH})_{2}\right]\left(\mathrm{A}^{n-}\right)_{x / n} \cdot m \mathrm{H}_{2} \mathrm{O}$, where $\mathrm{M}^{2+}$ and $\mathrm{M}^{3+}$ are di- and trivalent metal cations, and $\mathrm{A}^{n-}$ is a counter anion. ${ }^{8,9}$ Recently, LDHs containing transition metal elements have been reported to be promising electrode materials for supercapacitors because of their relatively low cost, high redox activity, environmentally friendly nature and effective utilization of transition metal atoms. ${ }^{\mathbf{1 0}}$ For instance, Wang's group found that electrodes consisting of NiAl-

\footnotetext{
a State Key Laboratory of Chemical Resource Engineering, Beijing University of Chemical Technology, Beijing 100029, China. E-mail: sunxm@mail.buct.edu.cn; Fax: +86-10-64425385; Tel: +86-10-64448751

${ }^{b}$ Chemistry Research Laboratory, Department of Chemistry, University of Oxford, Mansfield Road, Oxford, UK OX1 3TA

'School of Human Sciences, London Metropolitan University, Holloway Road, London, UK N7 8DB

$\uparrow$ Electronic supplementary information (ESI) available: Detailed experimental procedure, specific capacitance calculation, EDS and FTIR results, electrochemical results of CoAl-LDH and SEM image. See DOI: $10.1039 / \mathrm{c} 2 \mathrm{nr} 30617 \mathrm{~d}$
}

LDH coated on the surface of nickel foam and NiAl-LDH-graphene composites could display specific capacitances of $701 \mathrm{~F} \mathrm{~g}^{-1}$ and 785 $\mathrm{F} \mathrm{g}^{-1}$, respectively. ${ }^{10,11}$ More recently, Jin et al. developed CoAl-LDH and GO composites as high-performance supercapacitive materials. ${ }^{12}$ Although LDHs have been investigated widely, their electrochemical behavior is usually not good enough to meet the demand of new energy storage devices. The limited performance is mainly attributed to the restriction of the architecture and structure.

Constructing the active material into nanoarray architectures could improve electrochemical performance further, because these architectures ${ }^{13,14}$ could prevent agglomeration, facilitate fast electron transfer and ensure that all the individual crystallization participated in the electrochemical reaction. ${ }^{15}$ These investigations were mainly based on mesoporous transition metal oxides. ${ }^{16-18}$ There exist porous metal carbonate hydroxides generated using sacrificial polystyrene (PS) colloidal crystal templates, ${ }^{19}$ but the complicated preparation procedure and high cost have limited industrial production. Therefore, a simple, low cost and environmentally friendly method for the preparation of metal carbonate or hydroxides with mesoporous structures is sought after to deliver scalable and high-performance electrodes.

Herein, we report a facile method to prepare a mesoporous cobalt carbonate hydroxide thin nanosheet array supercapacitor electrode with improved supercapacitance performance using a precursive CoAl-LDH nanosheet array. The maximum specific capacitance reached $1075 \mathrm{~F} \mathrm{~g} \mathrm{~g}^{-1}$ at $5 \mathrm{~mA} \mathrm{~cm}^{-2}$, and $780 \mathrm{~F} \mathrm{~g}^{-1}(\sim 72 \%)$ could be maintained at a much higher current density $\left(50 \mathrm{~mA} \mathrm{~cm}{ }^{-2}\right)$. Additionally, even after 2000 cycles of charge and discharge at $30 \mathrm{~mA}$ $\mathrm{cm}^{-2}, 92 \%$ of the initial capacitance can be preserved, indicating its high stability.

The precipitation and conversion process was schematically shown in Fig. 1. Firstly, a CoAl-LDH nanosheet array was synthesized by co-precipitation under hydrothermal conditions, which resulted in a pink coating on the surface of the nickel foam (inset of Fig. 2A).

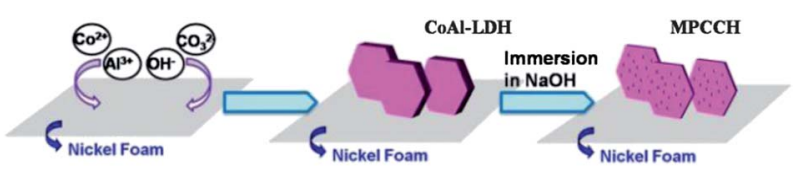

Fig. 1 Schematic illustration showing the fabrication of the MPCCH thin nanosheet array from a CoAl-LDH nanosheet array. 


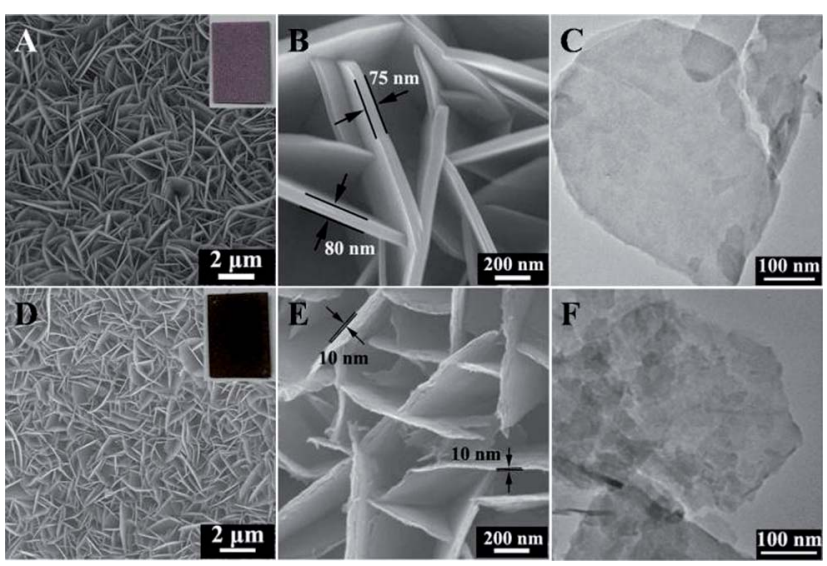

Fig. 2 Low (A) and high (B) magnification SEM images of the CoAlLDH nanosheet array (inset: an optical image of the CoAl-LDH nanosheet array). (C) presents a typical TEM image of the CoAl-LDH. Also provided are low (D) and high (E) magnification SEM images of the $\mathrm{MPCCH}$ thin nanosheet array (inset: an optical image of the MPCCH thin nanosheet array). (F) represents a typical TEM image of MPCCH.

Fig. 2A and B show SEM images of the as-synthesized CoAl-LDH nanosheet array taken at different magnifications. The SEM data revealed that the array consisted of regular hexagonal nanosheets, typically $0.5-1 \mu \mathrm{m}$ in length and $50-80 \mathrm{~nm}$ in thickness, arranged as a dense film on the nickel foam. The nanosheets grew vertically and were cross-linked on the Ni substrate. After immersion in $5 \mathrm{M} \mathrm{NaOH}$ solution, the color of the film changed to dark red (see inset of Fig. 2D). SEM results indicated that it preserved its 3D network and porous structure without collapse (see Fig. 2D), but the individual nanosheets became thinner (with thicknesses of $c a .10 \mathrm{~nm}$ ) after this treatment (Fig. 2E), and showed extensive cracking (e.g. at the edges and the centre). Small holes of several to tens of nanometres in size were clearly observable in the cracked nanosheets (Fig. 2F), in contrast to the well-crystallised CoAl-LDH nanosheets (Fig. 2C). Compared with the highly crystalline CoAl-LDH nanosheet array, the alkali treated array was expected to have a much higher specific surface area. The $\mathrm{Co}: \mathrm{Al}$ atomic ratio of the modified films was determined by energy-dispersive X-ray spectroscopy (EDS; see Fig. S1†). A Co : Al ratio of $c a .3: 1$ is calculated for the initial CoAlLDH electrode (Fig. S1A $\dagger$ ), whereas only Co can be observed in the basic etched electrode (Fig. $\mathrm{S} 1 \mathrm{~B} \dagger$ ), which indicates that the Al had been almost extracted from these films by the highly concentrated $\mathrm{NaOH}$ solution. Combining the EDS, SEM and TEM data it was concluded that etching in the concentrated basic solution led to the extraction of $\mathrm{Al}$ cations from the CoAl-LDH and the formation of holes and cracks in the nanosheets, which resulted in mesoporous cobalt carbonate hydroxide (MPCCH) nanosheets. The process thinned the sheets, increased the surface area, and facilitated the penetration of electrolytes into electrodes.

In contrast to the significant change in morphology, the extraction of $\mathrm{Al}$ from the $\mathrm{LDH}$ precursor did not cause significant changes in the crystal structure. The XRD patterns of the CoAl-LDH and MPCCH films were shown in Fig. 3. XRD data of the CoAl-LDH film showed a series of Bragg reflections in good agreement with the well-known $\mathrm{LDH} \mathrm{Co}_{6} \mathrm{Al}_{2} \mathrm{CO}_{3}(\mathrm{OH})_{16} \cdot \mathrm{H}_{2} \mathrm{O}$ (JCPDF: 51-0045; the peaks marked "\#" denote the Ni substrate). No Bragg reflections due to crystalline impurities were observed. The observed interlayer

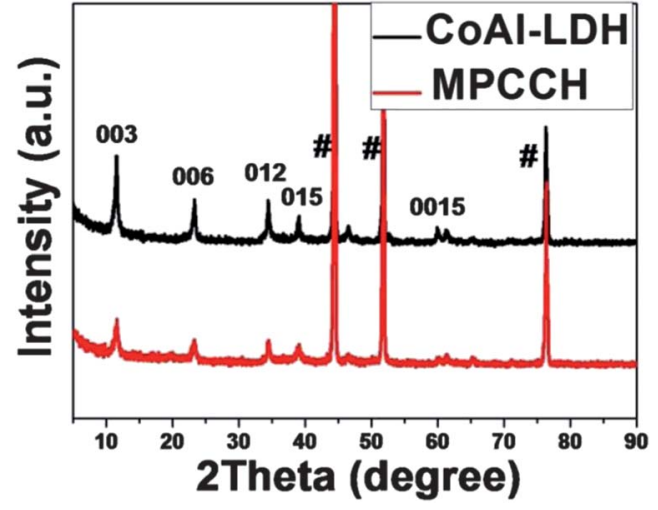

Fig. 3 XRD patterns of the CoAl-LDH (black line) and MPCCH nanosheet arrays (red line). Reflections marked \# correspond to the $\mathrm{Ni}$ foam on which the thin films are grown.

separation of $0.75 \mathrm{~nm}\left(d_{003}\right)$ indicated that $\mathrm{CO}_{3}{ }^{2-}$ ions and water molecules occupied the interlayer galleries, which was confirmed by FT-IR data (see Fig. S2 $\dagger$ ). The XRD pattern for the MPCCH (Fig. 3, red line) still exhibited Bragg reflections at the same $2 \theta$ values as the $\mathrm{CoAl}-\mathrm{CO}_{3} \mathrm{LDH}$, indicating that the layer structure was retained. However, the overall intensity of the XRD pattern had decreased while the relative intensities of the Bragg reflections were unchanged. The peak widths at half maximum had increased, indicative of decreased crystallinity.

The MPCCH thin nanosheet array was characterized by cyclic voltammetry $(\mathrm{CV})$, electrochemical impedance spectroscopy (EIS), and galvanostatic charge/discharge measurements to assess its potential for supercapacitor electrodes. The CoAl-LDH nanosheet array was also investigated as a comparison. Fig. 4A showed the cyclic voltammogram of the MPCCH array at scan rates of 1,5 and $10 \mathrm{mV} \mathrm{s}^{-1}$ with a potential range of $0-0.5 \mathrm{~V}$ (vs. SCE) in $2 \mathrm{M} \mathrm{NaOH}$

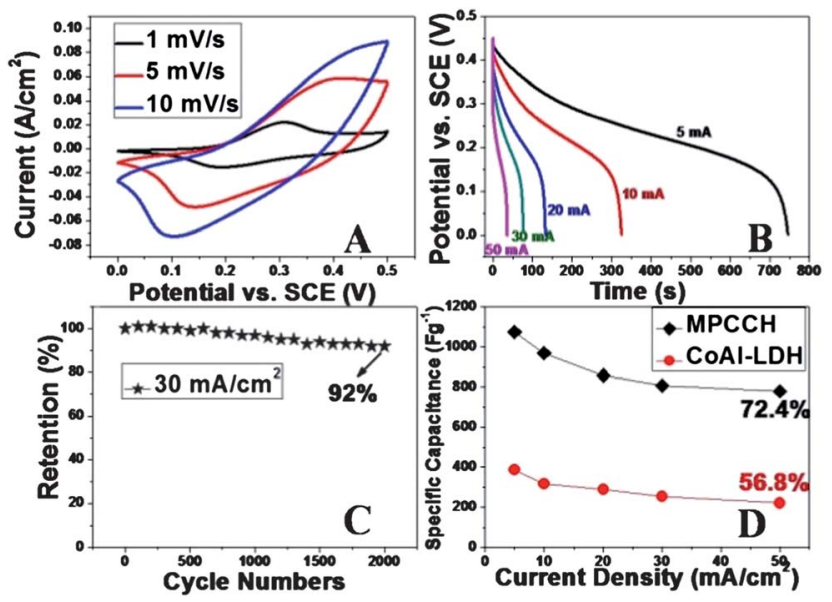

Fig. 4 Electrochemical characterization of the MPCCH thin nanosheet array. (A) CV curves of the MPCCH thin nanosheet array at different scan rates $\left(1-10 \mathrm{mV} \mathrm{s}^{-1}\right)$; (B) galvanostatic discharge curves of the MPCCH at various discharge current densities $\left(5-50 \mathrm{~mA} \mathrm{~cm}{ }^{-2}\right)$; (C) specific capacitance retention versus cycle number of the MPCCH at a galvanostatic charge and discharge current density of $30 \mathrm{~mA} \mathrm{~cm}^{-2}$; (D) comparison of the average specific capacitance versus charge and discharge current density between MPCCH and CoAl-LDH. 
solution. For each curve, the set of redox peaks observed indicated the existence of a faradic process which was ascribed to the interconversion of $\mathrm{Co}(\mathrm{II}) / \mathrm{Co}(\mathrm{III})$, as previous research on CoAl-LDH had indicated. ${ }^{20}$ Fig. 4B gave the results obtained for the MPCCH thin nanosheet array electrode over the potential range of $0-0.45 \mathrm{~V}$ at various current rates $\left(5,10,20,30\right.$ and $\left.50 \mathrm{~mA} \mathrm{~cm}^{-2}\right)$. The specific capacitance was calculated to be $1075 \mathrm{~F} \mathrm{~g}^{-1}\left(8.6 \mathrm{~F} \mathrm{~cm}^{-2}\right)$ at a low current density $\left.(5 \mathrm{~mA} \mathrm{~cm})^{-2}\right)$, and $780 \mathrm{~F} \mathrm{~g}^{-1}\left(6.24 \mathrm{~F} \mathrm{~cm}^{-2}\right)$ at a very high current density $\left(50 \mathrm{~mA} \mathrm{~cm}{ }^{-2}\right)$. Hence, more than $72 \%$ of the capacitance was maintained at elevated current densities (Fig. 4D, dark line), indicating an excellent rate capability.

Cycling performance is another key factor which must be considered in developing supercapacitor electrodes for practical applications. The cycle charge/discharge testing was employed to examine the cycling stability of the MPCCH electrode at a current density of $30 \mathrm{~mA} \mathrm{~cm}^{-2}$ (Fig. 4C). After 2000 cycles, the capacitance remains at $92 \%$ of its initial value. This demonstrated that, within the voltage window $0.0-0.45 \mathrm{~V}$, repeated charge and discharge processes did not seem to induce significant structural changes to the MPCCH thin nanosheet electrode, as expected for pseudo-capacitance processes. The SEM image of the MPCCH thin nanosheet array (Fig. S3†) retained its $3 \mathrm{D}$ network structure after repeated charge/discharge cycles. The combination of high specific capacitance, excellent kinetics and long-term stability implied that the $\mathrm{MPCCH}$ thin nanosheet arrays could be used as an advanced electrode in supercapacitors.

We carried out similar electrochemical measurements on the precursor CoAl-LDH hexagonal nanosheet array (Fig. S4A and $\mathrm{B} \dagger$ ). Fig. $\mathrm{S} 4 \mathrm{C} \uparrow$ clearly showed that the resulting current from $\mathrm{MPCCH}$ was much higher than that of the CoAl-LDH films at the same scan rate, resulting in a larger area in the CV curve of the MPCCH electrode. This indicated that the MPCCH thin nanosheet array exhibited a much higher specific capacitance than the LDH electrode. Fig. 4D summarized the specific capacitance at different current densities calculated from the discharge curves, and demonstrated that the MPCCH electrode yielded an enhanced capacitance performance, with a factor of $\sim 1.5$ increase in specific capacitance compared to the CoAl-LDH electrode. Furthermore, the capacitance of the CoAl-LDH nanosheet array was decreased to $220 \mathrm{~F} \mathrm{~g}^{-1}$ upon an increase in current density to $30 \mathrm{~mA} \mathrm{~cm}^{-2}$ (56.8\% of the capacitance at lower current density), indicating weaker rate capability. Electrochemical impedance spectroscopy results (EIS, Fig. S5†) further demonstrated that the MPCCH thin nanosheet array provided lower resistance and smaller charge transfer resistance, which was also beneficial to the rate capability of the electrode. ${ }^{15}$ Our data indicated that the capacitive behavior of the MPCCH thin nanosheet array was significantly improved compared to the corresponding CoAl-LDH precursor. Therefore, we believe that after basic treatment the MPCCH electrode had an increased contact area between the electrode and the electrolyte, resulting in more efficient utilization of the active material. The cycling stability was comparable with that reported, ${ }^{10,12}$ but the specific capacitance was higher than that of previously reported LDH-like pseudocapacitive materials including CoAl-LDH, NiAl-LDH and their composites with graphene (700-900 $\left.\mathrm{F} \mathrm{g}^{-1}\right)$. $^{10,11,20,21}$ The rate capability was even higher than that of the composite of CoAl-LDH and graphene, indicating the advantage of its mesoporous structure. ${ }^{12}$

Understanding the origins of the high-performance capacitive behavior, including high specific capacitance, excellent rate capability, and long-term cycling stability, was important in order to fabricate better and more practical devices. Since both MPCCH and its LDH precursor had a 3D network and array-like architecture, uniform crystallization size without agglomeration, and tight binding between the active material and the substrate (or current collector), ${ }^{22,23}$ the higher capacitive performance of the MPCCH electrode as compared with the CoAl-LDH electrode was attributed to the high porosity generated from the "holes" in the structure and the reduced thickness of individual sheets. Compared with an intact nanosheet, the holey mesoporous nanosheet exposed a larger contact area to the electrolyte and provided abundant $\mathrm{OH}^{-}$ion binding sites, resulting in enhanced ion diffusion kinetics. ${ }^{24}$ The thin nature of the $\mathrm{MPCCH}$ nanosheets was another important feature of the MPCCH electrode, as the electrolyte had a penetration depth of approximately $20 \mathrm{~nm} .^{25}$ Hence, the very thin $(\sim 10 \mathrm{~nm}) \mathrm{MPCCH}$ sheets effectively shortened the proton diffusion distance and made more chemically active material available for the redox reaction. BET (Brunauer-EmmetTeller) results indicated that MPCCH arrays had a higher surface area per centimetre of $\mathrm{Ni}$ foam $\left(0.502 \mathrm{~m}^{2} \mathrm{~cm}^{-2}\right)$ than the CoAl-LDH array $\left(0.365 \mathrm{~m}^{2} \mathrm{~cm}^{-2}\right)$, which was in accordance with our hypothesis. Therefore, the larger capacitance for the MPCCH thin nanosheet array can be easily understood by the combination of electric doublelayer capacitance generated by larger surface area and faradic pseudocapacitance resulting from efficient utilization of the electrode material.

In summary, a simple, low-cost, template-free and environmentally friendly method has been developed to fabricate a thin mesoporous cobalt carbonate hydroxide nanosheet array by immersing a CoAl$\mathrm{LDH}$ thin film in highly concentrated $\mathrm{NaOH}$ solution. The unique structure shows superior electrochemical performance (a maximum capacitance of $1075 \mathrm{~F} \mathrm{~g} \mathrm{~g}^{-1}$ at $5 \mathrm{~mA} \mathrm{~cm} \mathrm{~cm}^{-2}$, with almost $72 \%$ retention at a very high current density of $50 \mathrm{~mA} \mathrm{~cm}^{-2}$, and more than $92 \%$ of the capacitance preserved after 2000 charge/discharge cycles) compared with a CoAl-LDH nanosheet array electrode. The excellent performance is attributed to the mesoporous structure and reduced thickness of the MPCCH nanosheets. The fabrication approach described in this work should be applicable for the synthesis of other mesoporous metal carbonates or hydroxides from LDH precursors.

This work was financially supported by the NSFC, the 973 Program (no. 2011CBA00503, 2011CB932403), the 863 Program (grant no. 2012AA03A609), the Program for New Century Excellent Talents in Universities, and the Foundation for Authors of National Excellent Doctoral Dissertations of P. R. China.

\section{Notes and references}

1 P. Simon and Y. Gogotsi, Nat. Mater., 2008, 7, 845-854.

2 C. Liu, F. Li, L. P. Ma and H. M. Cheng, Adv. Mater., 2010, 22, E28E62.

3 H. L. Wang, H. S. Casalongue, Y. Liang and H. J. Dai, J. Am. Chem. Soc., 2010, 132, 7472-7477.

4 G. Yu, L. Hu, N. Liu, H. Wang, M. Vosgueritchian, Y. Yang, Y. Cui and Z. Bao, Nano Lett., 2011, 11, 4438-4442.

5 J. K. Chang, C. M. Wu and I. W. Sun, J. Mater. Chem., 2010, 20, 3729-3735.

6 M. Kaempgen, C. K. Chan, J. Ma, Y. Cui and G. Gruner, Nano Lett., 2009, 9, 1872-1876.

7 J. Huang, V. Meunier, B. G. Sumpter, P. M. Ajayan, J. J. Yoo, K. Balakrishnan, A. Srivastava, M. Conway, A. L. M. Reddy and J. Yu, Nano Lett., 2011, 11, 1423-1427.

8 A. I. Khan and D. O'Hare, J. Mater. Chem., 2002, 12, 3191-3198.

9 G. R. Williams and D. O'Hare, J. Mater. Chem., 2006, 16, 3065-3074. 
10 Z. Gao, J. Wang, Z. Li, W. Yang, B. Wang, M. Hou, Y. He, Q. Liu, T. Mann and P. Yang, Chem. Mater., 2011, 23, 3509-3516.

11 J. Wang, Y. Song, Z. Li, Q. Liu, J. Zhou, X. Jing, M. Zhang and Z. Jiang, Energy Fuels, 2010, 24, 6463-6467.

12 L. Wang, D. Wang, X. Y. Dong, Z. J. Zhang, X. F. Pei, X. J. Chen, B. Chen and J. Jin, Chem. Commun., 2011, 47, 3556-3558.

13 Y. Li, B. Tan and Y. Wu, Nano Lett., 2008, 8, 265-270.

14 Y. Yang, D. Kim, M. Yang and P. Schmuki, Chem. Commun., 2011, 47, 7746-7748.

15 J. Liu, J. Jiang, C. Cheng, H. Li, J. Zhang, H. Gong and H. J. Fan, Adv. Mater., 2011, 23, 2075-2080.

16 X. W. Lou, D. Deng, J. Lee, J. Feng and L. A. Archer, Adv. Mater., 2008, 20, 258-262.

17 H. Wang, L. Zhang, X. Tan, C. M. B. Holt, B. Zahiri, B. C. Olsen and D. Mitlin, J. Phys. Chem. C, 2011, 115, 17599-17605.
18 D. Wang, Q. Wang and T. Wang, Inorg. Chem., 2011, 50, 6482-6492.

19 E. Géraud, S. Rafqah, M. Sarakha, C. Forano, V. Prevot and F. Leroux, Chem. Mater., 2007, 20, 1116-1125.

20 L. Zhang, X. Zhang, L. Shen, B. Gao, L. Hao, X. Lu, F. Zhang, B. Ding and C. Yuan, J. Power Sources, 2012, 199, 395-401.

21 Y. Wang, W. Yang, C. Chen and D. G. Evans, J. Power Sources, 2008, 184, 682-690.

22 Z. Y. Lu, Z. Chang, J. F. Liu and X. M. Sun, Nano Res., 2011, 4, 658665.

23 Z. Y. Lu, Z. Chang, W. Zhu and X. M. Sun, Chem. Commun., 2011, 47, 9651-9653.

24 X. Zhao, C. M. Hayner, M. C. Kung and H. H. Kung, ACS Nano, 2011, 5, 8739-8749.

25 C. C. Hu, K. H. Chang, M. C. Lin and Y. T. Wu, Nano Lett., 2006, 6, 2690-2695. 